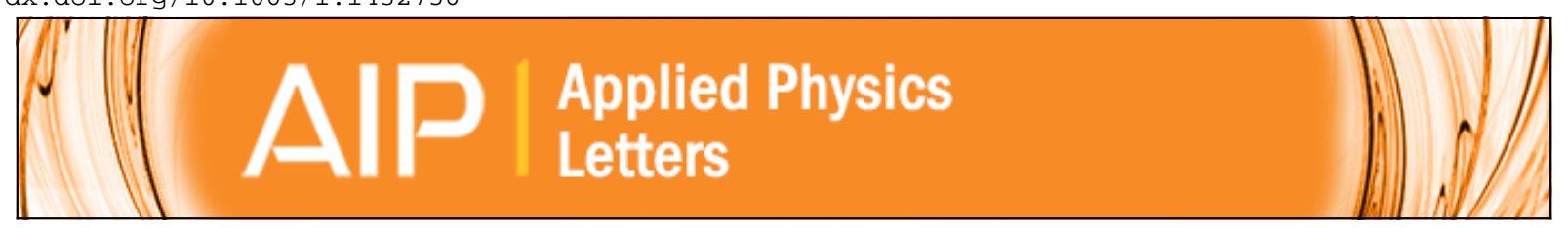

Two-photon-excited green emission and its dichroic shift of oriented thin-film CdS on glass formed by laser deposition

B. Ullrich, R. Schroeder, H. Sakai, A. Zhang, and S. Z. D. Cheng

Citation: Applied Physics Letters 80, 356 (2002); doi: 10.1063/1.1432756

View online: http://dx.doi.org/10.1063/1.1432756

View Table of Contents: http://scitation.aip.org/content/aip/journal/apl/80/3?ver=pdfcov

Published by the AIP Publishing

Over 700 papers \&

presentations on

multiphysics simulation visw now

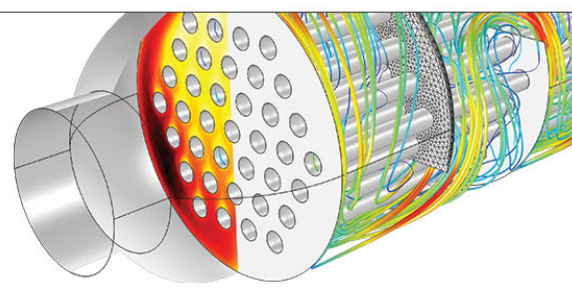




\title{
Two-photon-excited green emission and its dichroic shift of oriented thin-film CdS on glass formed by laser deposition
}

\author{
B. Ullrich ${ }^{\mathrm{a})}$ and R. Schroeder ${ }^{\mathrm{b})}$ \\ Department of Physics and Astronomy, Centers for Materials Science and Photochemical Sciences, \\ Bowling Green State University, Bowling Green, Ohio 43403-0224 \\ H. Sakai \\ Faculty of Engineering, Hiroshima Kokusai Gakuin University, 6-20-1 Nakao, Aki-ku, Hiroshima 739-0321, \\ Japan
}

\author{
A. Zhang and S. Z. D. Cheng \\ Maurice Morton Institute and Department of Polymer Science, Goodyear Polymer Center, \\ University of Akron, Akron, Ohio 44325-3909
}

(Received 17 September 2001; accepted for publication 31 October 2001)

\begin{abstract}
The photoluminescence of oriented thin-film $\mathrm{CdS}$ on glass formed by laser deposition was investigated employing $200 \mathrm{fs}, 1.54 \mathrm{eV}$ laser pulses at room temperature. The ultrafast excitation caused a two-photon absorption process, which results in purely green emission at the band gap. The spectra are fitted very well by the application of the van Roosbroeck-Shockley relation, density of states, and Urbach's rule demonstrating the intrinsic character of the radiative recombination. It is further shown that the energy position of the emission peak depends on the polarization of the impinging laser beam due to the dichroism of the highly oriented films. (C) 2002 American Institute of Physics. [DOI: 10.1063/1.1432756]
\end{abstract}

Modern photonics and optoelectronics address the relevant problem for many device applications of obtaining stable sources of green photoluminescence. ${ }^{1}$ Eye adapted light emission devices (LED's) are of particular interest. Thin-film devices based on the II-VI compound semiconductor CdS appear to be a promising concept for adapted LED's since the expected band gap emission at about 2.45 $\mathrm{eV}$ at room temperature lies very close to the highest sensitivity of the human eye. ${ }^{2}$ Indeed, in previous articles, ${ }^{3,4}$ it was demonstrated that thin-film $\mathrm{CdS}$ on glass formed by pulsed-laser deposition (PLD) at $1064 \mathrm{~nm}$ exhibit green light emission and lasing at $2.49 \mathrm{eV}$ at room temperature. The films crystallize in a wurtzite structure and the $\mathbf{c}$ axis, which runs along the $\langle 001\rangle$ direction, is perpendicularly oriented with respect to the glass surface independently of the incident laser intensity (laser fluence) applied for the PLD. On the other hand, CdS films on glass formed by PLD at $355 \mathrm{~nm}$ show different features from the crystallographic and optoelectronic points of view. The films also exhibit a wurtzite structure but the orientation of the $\langle 001\rangle$ direction depends on the laser fluence applied to form the film; at $2 \mathrm{~J} \mathrm{~cm}^{-2}$ and $\geqslant 4 \mathrm{~J} \mathrm{~cm}^{-2}$, the $\langle 001\rangle$ direction is perpendicular and parallel to the glass surface, hereafter referred to $\mathrm{CdS}_{\perp}$ and $\mathrm{CdS}_{\|}$, respectively. The details about the formation of oriented thinfilm CdS by PLD have been published in previous articles. ${ }^{5,6}$ The photocurrent and transmission spectra measured with polarized light reflect the dichroism of the films by shifting the onset of the interband absorption of $\mathrm{CdS}_{\perp} 55 \mathrm{meV}$ below that of $\mathrm{CdS}_{\|} .{ }^{7}$ The single-photon photoluminescence (SPL) excited with the continuous wave $325 \mathrm{~nm}$ line of a $\mathrm{He}-\mathrm{Cd}$

${ }^{a)}$ Electronic mail: bruno@kottan-labs.bgsu.edu

${ }^{b)}$ Also at: Department of Physics, Virginia Tech, Blacksburg, Virginia 24061-0435. laser, however, does not reflect the dichroism of the films and shows an opposite shift in energy. The emission peak of $\mathrm{CdS}_{\perp}$ and $\mathrm{CdS}_{\|}$centers at $2.45 \mathrm{eV}$ and $2.27 \mathrm{eV}$, respectively. ${ }^{8}$

In this letter, we demonstrate that the dichroism of oriented thin-film CdS is reflected by the emission properties if the bulk is excited. For this purpose two-photon excitation in the femtosecond regime at $1.54 \mathrm{eV}$ was used.

The samples investigated were prepared by PLD by guiding the $355 \mathrm{~nm}$ emission of Nd:YAG laser (pulse width $5 \mathrm{~ns}$, repetition rate $10 \mathrm{~Hz}$ ) onto a target of sintered $\mathrm{CdS}$ (99.999\%) powder. The distance between the target and the fused silica glass substrate was $3 \mathrm{~cm}$. During the PLD process, the substrate temperature and ambient pressure were kept at $250^{\circ} \mathrm{C}$ and $10^{-3} \mathrm{~Pa}$, respectively. The samples were formed with laser fluences of $2 \mathrm{~J} \mathrm{~cm}^{-2}$ and $5 \mathrm{~J} \mathrm{~cm}^{-2}$, resulting in $\mathrm{CdS}_{\perp}$ and $\mathrm{CdS}_{\|}$as demonstrated by the x-ray patterns in Fig. 1. We should stress that the possibility to control the dichroism of thin-film CdS is interesting especially for the fabrication of photonic structures with a periodic dielectric structure. $^{9,10}$ By means of transmission spectroscopy, the thickness of the films was found to be $1.70 \mu \mathrm{m}$ and $0.82 \mu \mathrm{m}$ for $\mathrm{CdS}_{\perp}$ and $\mathrm{CdS}_{\|}$, respectively.

The photoluminescence of the films was excited by an ultrafast laser system, which delivers 200 fs pulses with a repetition rate of $249 \mathrm{kHz}$ at $1.54 \mathrm{eV}(804 \mathrm{~nm})$ and an energy per pulse of $1.5 \mu \mathrm{J}$. The beam was focused to an $e^{-2}$ radius of $\approx 30 \mu \mathrm{m}$ resulting in an intensity of $200 \mathrm{GW} \mathrm{cm}{ }^{-2}$. The laser beam exhibits linear horizontal polarization and therefore by placing $\mathrm{CdS}_{\perp}$ and $\mathrm{CdS}_{\|}$in the beam, the direction of the electric field vector is perpendicular $(\mathbf{E} \perp \mathbf{c})$ and parallel $(\mathbf{E} \| \mathbf{c})$ to the $\mathbf{c}$ axis. The excitation caused clearly visible green light emission and was detected by a fiber optics spectrometer placed behind the sample. The emission intensity depends on the square of the impinging intensity revealing 


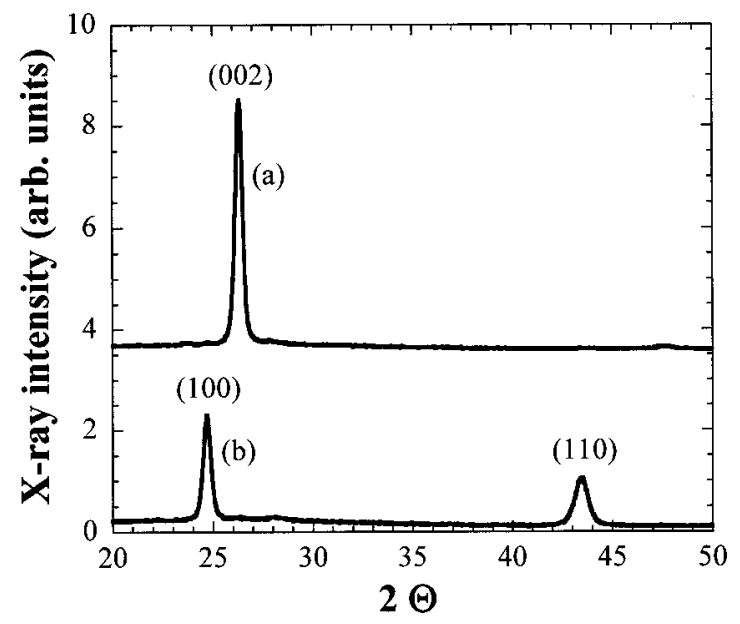

FIG. 1. X-ray patterns of (a) $\mathrm{CdS}_{\perp}$ and (b) $\mathrm{CdS}_{\|}$. The curves are shifted and plotted in the same arbitrary units.

the two-photon character of the phenomenon. Figures 2(a) and 2(b) show the photoluminescence spectrum due to twophoton excitation (TPL) of $\mathrm{CdS}_{\perp}$ and $\mathrm{CdS}_{\|}$. The symbols show the experimental data and the solid lines the fit according to the van Roosbroeck-Shockley relation, i.e., the principle of detailed balance, ${ }^{11}$

$$
I(h \nu) \propto \frac{(h \nu)^{2} \alpha(h \nu)}{\exp \left(h \nu / k T_{\mathrm{c}}\right)-1},
$$

where $h \nu$ is the photon energy, $\alpha$ is the absorption coefficient, $k$ is the Boltzmann constant, and $T_{\mathrm{c}}$ is the carrier temperature. The absorption coefficient versus energy is calculated by the density of states and Urbach's rule, and is given by $^{3}$

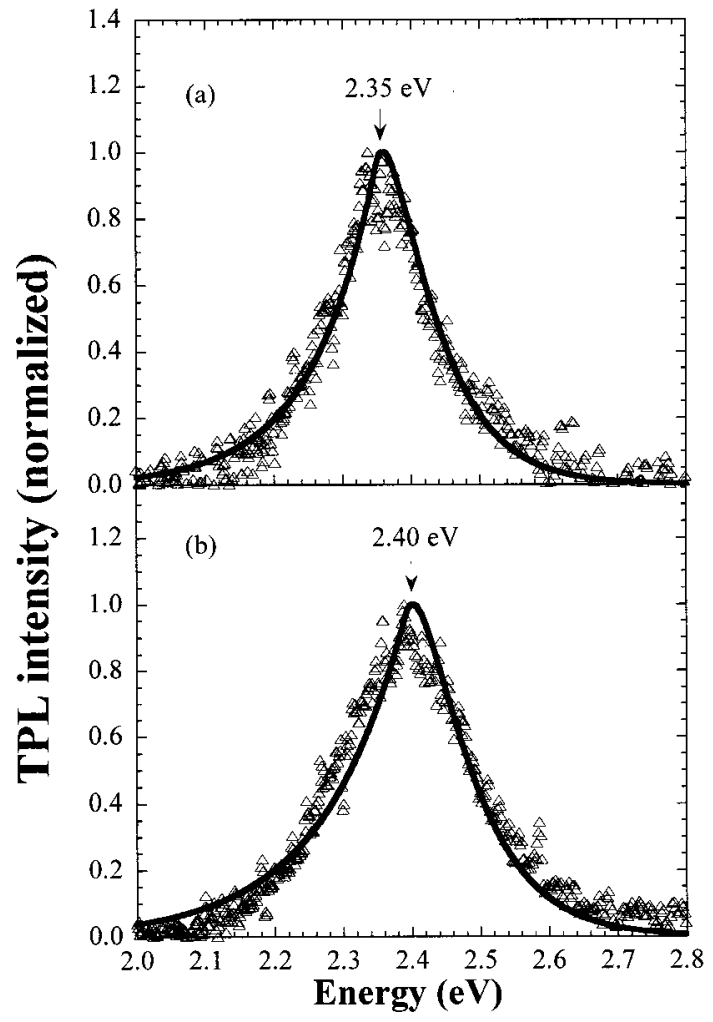

FIG. 2. TPL spectra of (a) $\mathrm{CdS}_{\perp}$ and (b) $\mathrm{CdS}_{\|}$at $300 \mathrm{~K}$.

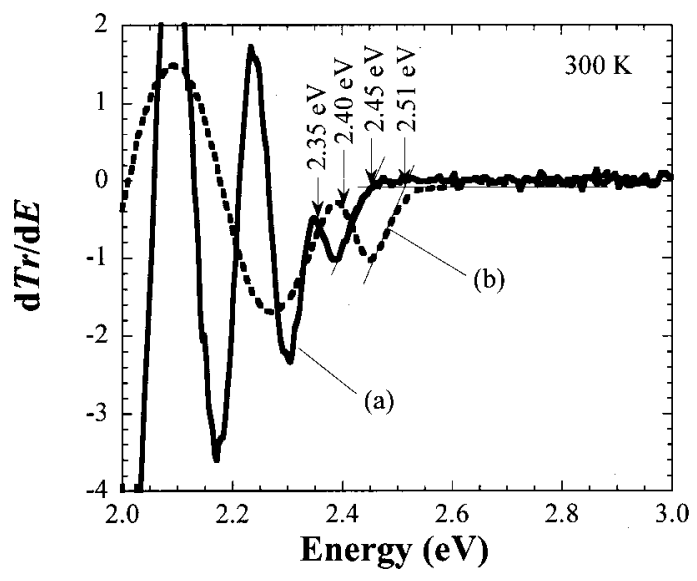

FIG. 3. $d T r / d E$ of (a) $\mathrm{CdS}_{\perp}$ and (b) $\mathrm{CdS}_{\|}$.

$$
\alpha(h \nu)=A\left(h \nu-E_{g}\right)^{1 / 2} \text { if } h \nu \geqslant E_{\mathrm{cr}}
$$

and

$$
\alpha(h \nu)=A \sqrt{\frac{k T}{2 \sigma}} \exp \left\{\frac{\sigma}{k T}\left(h \nu-E_{\mathrm{cr}}\right)\right\} \quad \text { if } h \nu \leqslant E_{\mathrm{cr}},
$$

where $E_{g}$ is the band gap energy, $A=2 \times 10^{5} \mathrm{~cm}^{-1}(\mathrm{eV})^{-1 / 2}$ represents $\alpha(h \nu)$ for $h \nu \gg E_{g}, T$ is the lattice temperature, and the crossover energy between Eqs. (2) and (3) is expressed by $E_{\mathrm{cr}}=E_{g}+k T / 2 \sigma$. The dimensionless phenomenological parameter $\sigma$ is a measure for the pureness of the material. ${ }^{3}$ For undoped intrinsic CdS, it is generally accepted that $\sigma=2.17$. Numbers below 2.17 are typical for thin-film $\mathrm{CdS}$ and indicate tail states beyond the intrinsic density. The following parameters were used to fit the data for $\mathrm{CdS}_{\perp}$ in Fig. 2(a), $E_{g}=2.33 \mathrm{eV}, k T=25 \mathrm{meV}, k T_{\mathrm{c}}=55 \mathrm{meV}$, and $\sigma=0.7$, and for $\mathrm{CdS}_{\|}$in Fig. 2(b), $E_{g}=2.37 \mathrm{eV}, k T=25 \mathrm{meV}$, $k T_{\mathrm{c}}=60 \mathrm{meV}$, and $\sigma=0.6$. As it is known from photoluminescence investigations on GaAs, the carriers attain a temperature much higher than that of the lattice. ${ }^{12}$ It is noteworthy that the emission takes place according to the straightforward principle of detailed balance since $\mathrm{CdS}$ is known to show peculiar emission features far from the ideal case. $^{13}$

As shown in Figs. 2(a) and 2(b), the TPL peak is at 2.35 $\mathrm{eV}$ and $2.40 \mathrm{eV}$ for $\mathrm{CdS}_{\perp}$ and $\mathrm{CdS}_{\|}$, respectively. In order to check if the energy shift of $50 \mathrm{meV}$ is subject to the dichroism corresponding to the polarizations $\mathbf{E} \perp \mathbf{c}$ and $\mathbf{E} \| \mathbf{c}$, it is of importance to calculate the penetration depth of the fs-laser beam,

$$
d_{p}=\frac{1}{\beta I_{0}}(e-1),
$$

where $\beta(=6.4 \mathrm{~cm} / \mathrm{GW})^{14}$ is the two-photon absorption coefficient and $I_{0}$ is the impinging laser intensity $(=200$ $\mathrm{GW} \mathrm{cm}{ }^{-2}$ ), and find $d_{p}=13 \mu \mathrm{m}$. Since the film thicknesses are clearly shorter than $d_{p}$, the samples are homogeneously excited and the spectra in Figs. 2(a) and 2(b) represent the bulk emissions of the samples. The curves of Figs. 3(a) and 3(b) show the first derivative of the transmittance $(T r)$ of $\mathrm{CdS}_{\perp}$ and $\mathrm{CdS}_{\|}$with respect to energy $(d T r / d E)$. As indicated, the $\operatorname{Tr}$ threshold of $\mathrm{CdS}_{\perp}$ and $\mathrm{CdS}_{\|}$takes place at 2.45 $\mathrm{eV}$ and $2.51 \mathrm{eV}$, respectively. Hence, in good agreement with our previous results shown in Reîs. 7 and 8 , and with the 
dichroism of single crystal $\mathrm{CdS}$ in the high absorptive region, ${ }^{15}$ the dichroic shift of the onset of the fundamental interband transition is $60 \mathrm{meV}$. The energy positions of the TPL peaks in Figs. 2(a) and 2(b) closely coincide with the first local maxima at the low energy side of the $d T r / d E$ spectra. These maxima mark the start of the Fabry-Perot fringes, i.e., the energy from where the films become sufficiently transparent such that reflections from the rear at the $\mathrm{CdS} /$ glass interface are not reabsorbed. As a consequence, the maxima of the bulk emissions in Figs. 2(a) and 2(b) are shifted to these energies below the band gap due to selfabsorption and their separation by $50 \mathrm{meV}$ is caused by the dichroism of the films. The shift is smaller than the dichroic separation in Fig. 3 since the bulk emission reflects the dichroic displacement of the absorption edge influenced by Urbach's tail rather than by the onset of the interband transition. Nevertheless, it is worthwhile to stress at this point that the $50 \mathrm{meV}$ shift in Fig. 2 exceeds the separation of photoluminescence manifolds due to crystal-field splitting ${ }^{16}$ in $\mathrm{GaN}$ doped with $\mathrm{Pr}$ and makes oriented thin-film $\mathrm{CdS}$ interesting for optoelectronic devices based on polarizing effects.

The aforementioned SPL peak of $\mathrm{CdS}_{\perp}$ at $2.45 \mathrm{eV}$ coincides with the $T r$ onset of $\mathrm{CdS}_{\perp}$ in Fig. 3 since SPL does not represent bulk features due to the restricted penetration depth of the excitation $(1 / \alpha=0.1 \mu \mathrm{m})$. The intrinsic features of $\mathrm{CdS}_{\|}$do not appear in the SPL due to the influence of traps and grain boundaries. ${ }^{8}$ Therefore, the comparison of SPL and TPL shows that TPL reveals the intrinsic features of the films independent of imperfections. This fact was confirmed by the investigation of $\mathrm{CdS}$ films formed by spray pyrolysis on pyrex ${ }^{\circledR} .{ }^{17}$ The SPL was visually dominated by red emission. The TPL however, exhibits exclusively green band gap photoluminescence of the bulk. We should stress that TPL evokes charge carrier densities of up to $10^{19} \mathrm{~cm}^{-3}$ within the pulse duration of $200 \mathrm{fs}^{17}$ This tremendous carrier density changes the emission behavior of the films in favor of the band gap emission.
In conclusion, excitation with 200 fs laser pulses at 1.54 $\mathrm{eV}$ causes room temperature single peak band gap emission in thin-film $\mathrm{CdS}_{\perp}$ and $\mathrm{CdS}_{\|}$due to two-photon absorption. The spectra are very well modeled by the principle of detailed balance. As a result, under high two-photon excitation, thin-film CdS formed by PLD can be considered as an intrinsic semiconductor. This observation is of considerable importance for the field of nonlinear optics since it demonstrates that excitation in the femtosecond regime reveals the intrinsic bulk features of the films, which are decisive in the fabrication of light emitting and laser devices. The work also stresses the possibility of PLD to form anisotropic CdS films. By comparing the emission spectra with the transmittance of the films, it was shown that the energy separation of $50 \mathrm{meV}$ between the TPL peaks of $\mathrm{CdS}_{\perp}$ and $\mathrm{CdS}_{\|}$is caused by the dichroic shift of the absorption edge.

${ }^{1}$ S. J. Matthews, Laser Focus World 36, 79 (2000).

${ }^{2}$ V. V. Sobolev, V. I. Donetskikh, and E. F. Zagainov, Sov. Phys. Semicond. 12, 646 (1978).

${ }^{3}$ B. Ullrich, D. M. Bagnall, H. Sakai, and Y. Segawa, Solid State Commun. 109, 757 (1999).

${ }^{4}$ D. M. Bagnall, B. Ullrich, X. G. Qiu, Y. Segawa, and H. Sakai, Opt. Lett. 24, 1278 (1999).

${ }^{5}$ H. Sakai, T. Tamaru, T. Sumomogi, H. Ezumi, and B. Ullrich, Jpn. J. Appl. Phys., Part 1 37, 4149 (1998).

${ }^{6}$ B. Ullrich, H. Sakai, N. M. Dushkina, H. Ezumi, S. Keitoku, and T. Kobayashi, Microelectron. Eng. 43-44, 695 (1998).

${ }^{7}$ B. Ullrich, J. W. Tomm, N. M. Dushkina, Y. Tomm, H. Sakai, and Y. Segawa, Solid State Commun. 116, 33 (2000).

${ }^{8}$ B. Ullrich, H. Sakai, and Y. Segawa, Thin Solid Films 385, 220 (2001).

${ }^{9}$ H. Kosaka, T. Kawashima, A. Tomita, M. Notomi, T. Tamamura, T. Sato, and S. Kawakami, Appl. Phys. Lett. 74, 1212 (1999).

${ }^{10}$ S. Noda, N. Yamamoto, M. Imada, H. Kobayashi, and M. Okano, J. Lightwave Technol. 17, 1948 (1999).

${ }^{11}$ W. van Roosbroeck and W. Shockley, Phys. Rev. 94, 1558 (1954).

${ }^{12}$ P. Y. Yu and B. Welber, Solid State Commun. 25, 209 (1978).

${ }^{13}$ X. W. Fan and J. Woods, Phys. Status Solidi A 70, 325 (1982).

${ }^{14}$ T. D. Krauss and F. W. Wiese, Appl. Phys. Lett. 65, 1739 (1994).

${ }^{15}$ D. Dutton, Phys. Rev. 112, 785 (1958).

${ }^{16}$ H. J. Lozykowski, W. M. Jadwisienczak, and I. Brown, J. Appl. Phys. 88, 210 (2000).

${ }^{17}$ B. Ullrich and R. Schroeder, Semicond. Sci. Technol. 16, L37 (2001). 